\title{
DESIGNING OPTIMAL WATER QUALITY MONITORING NETWORK FOR RIVER SYSTEMS AND APPLICATION TO A HYPOTHETICAL RIVER
}

\author{
Chuljin Park \\ Seong-Hee Kim \\ H. Milton Stewart School of \\ Industrial and Systems Engineering \\ Georgia Institute of Technology \\ Atlanta, GA 30332, USA
}

\author{
Ilker T. Telci \\ Mustafa M. Aral \\ School of Civil and Environmental Engineering \\ Georgia Institute of Technology \\ Atlanta, GA 30332, USA
}

\begin{abstract}
The problem of designing a water quality monitoring network for river systems is to find the optimal location of a finite number of monitoring devices that minimizes the expected detection time of a contaminant spill event with good detection reliability. We formulate this problem as an optimization problem with a stochastic constraint on a secondary performance measure where the primary performance measure is the expected detection time and the secondary performance measure is detection reliability. We propose a new objective function that integrates the stochastic constraint into the original objective function in a way that existing Optimization via Simulation (OvS) algorithms originally developed for an optimization problem without any stochastic constraint can be applicable to our problem. The performance of an OvS algorithm, namely the nested partitions method, with the new objective is tested on a hypothetical river.
\end{abstract}

\section{INTRODUCTION}

Maintaining good water quality for river systems is an important problem in environmental engineering. Many researchers have studied effects of contaminants, water purifying techniques, and systemized water quality monitoring. In this paper, we consider the problem of designing a water quality monitoring network for river systems where the goal is to find the optimal locations of a finite number of monitoring devices. The optimal network design should have the smallest expected detection time and high detection reliability. This problem is not easy due to hydrodynamics and contaminant transport in the river system and randomness in contaminant spill location and rain events. Also, the fact that we have two performance measures (detection time and reliability) and they are only observed through simulation makes the problem even more difficult.

Many researchers have studied water quality monitoring for river systems. For example, see the works of Beckers and Chamberlain (1974), Skalski and McKenzie (1982), Esterby, El-Shaarawi, and Block (1992) and Kwiatkowski (1991). Recent work includes Telci et al. (2008) and Telci et al. (2009). They studied the river network monitoring problem under hydrodynamics and contaminant transport with two objective functions, which are minimizing the detection time and maximizing the reliability for a hypothetical river and a real river (Altamaha River), respectively. However, they employed heuristic methods to find the best solution and considered a limited number of scenarios for contaminant spill and rainfall events with discrete distributions only.

The simulation community has developed a number of Optimization via Simulation (OvS) algorithms that find the best optimal or a good solution with global or local convergence guarantee when the objective function is evaluated through simulation and the optimization problem has deterministic constraints only. Among them, the nested partitions (NP) method (Shi and Ólafsson 2000) and the convergent optimization via most-promising-area stochastic search (COMPASS) (Hong and Nelson 2006) are representative frameworks for discrete optimization. NP is proven to converge the global optimal solution while COMPASS converges a local optimal solution. Although these algorithms are shown to perform well for many stochastic optimization problems, they are not directly applicable to our problem due to a stochastic constraint.

In this paper, we propose a new objective function that integrates a stochastic constraint into the original objective function in a way that existing OvS algorithms originally developed for an optimization problem 


\section{Park, Kim, Telci and Aral}

without any stochastic constraint can be applicable. The new objective function is called the penalized objective function. The value of the new objective function converges to the true mean of the original objective function for a feasible solution but diverges to infinity for an infeasible solution as the number of observations for the solution increases to infinity, assuming the problem is minimization. We apply an OvS algorithm with the penalized objective function to solve the water quality monitoring problems.

This paper is organized as follows: Section 2 details notation, outlines the problem formulation, and explain process simulation for hydrodynamics and contaminant transport in a river. Section 3 presents the penalized objective function and the NP method. Experimental results for steady state and dynamic flow cases in a hypothetical river are given in Section 4. Finally, Section 5 concludes the paper.

\section{PROBLEM DESCRIPTION}

In this section we introduce notation and formulate the problem of designing the water quality monitoring network. Then process simulation with hydrodynamics and contaminant transport is discussed.

\subsection{Notation and Problem Formulation}

The river network system has $N$ nodes (i.e., $N$ possible monitoring locations). Let $I$ be the index set and $I=\{1,2, \ldots, N\}$. The number of monitoring devices is $M$ and $M<N$. Each solution represents a location of $M$ devices and is denoted as an $M$ dimensional vector, $\mathbf{x}=\left[x_{1}, x_{2}, \ldots, x_{M}\right]^{T}$ such that $x_{i}, x_{j} \in I$ for $i, j=1, \ldots, M$ and $x_{i} \neq x_{j}$ for $i \neq j$. We also assume $x_{1}<x_{2}<\ldots<x_{M}$ to prevent the repetition of solutions (e.g. $(1,2,3)$ is the same as $(3,2,1),(2,1,3)$, etc). The whole solution set $\Theta$ is defined as the set of all possible $\mathbf{x}$.

Let $t_{d}\left(x_{i}\right)$ represent detection time at the monitoring location $x_{i}$ which is the first time when the concentration level at $x_{i}$ goes over the threshold constant $C_{t h}$. Then the elapsed detection time at $x_{i}, d\left(x_{i}\right)$ is defined as,

$$
d\left(x_{i}\right)= \begin{cases}t_{d}\left(x_{i}\right)-T_{s}, & \text { if a contaminant is detected at } x_{i} ; \\ P_{v}, & \text { otherwise }\end{cases}
$$

where $T_{S}$ is contaminant injection time (i.e., the starting time of the contaminant spill event) and $P_{v}$ is a large penalty constant for missing the contaminant spill detection. The minimum elapsed detection time for $\mathbf{x}, t(\mathbf{x})$, is defined as

$$
t(\mathbf{x})=\min _{1 \leq i \leq M} d\left(x_{i}\right)
$$

An indicator, $R(\mathbf{x})$ is,

$$
R(\mathbf{x})= \begin{cases}0, & \text { if none of monitors located at } \left.\mathbf{x} \text { detects a contaminant (i.e., } t(\mathbf{x})=P_{v}\right) \\ 1, & \text { otherwise (i.e., } \left.t(\mathbf{x})<P_{v}\right)\end{cases}
$$

With above two measurable functions and a required reliability level $0<q<1$, the main optimization formulation is as follows:

$$
\begin{aligned}
& \operatorname{argmin}_{\mathbf{x} \in \Theta} \mathbf{E}[t(\mathbf{x})] \\
& \text { subject to } \mathbf{E}[R(\mathbf{x})] \geq q .
\end{aligned}
$$

Note that $\mathbf{E}[t(\mathbf{x})]$ and $\mathbf{E}[R(\mathbf{x})]$ are estimated only through simulation. In next subsection, we explain how to estimate $\mathbf{E}[t(\mathbf{x})]$ and $\mathbf{E}[R(\mathbf{x})]$ using a process simulation with randomness in contaminant spill and rainfall events.

\subsection{Process Simulation}

To simulate hydrodynamics and contaminant transport in the river system, the EPA Storm Water Management Model (SWMM) is used. As in the user manual (U.S. Environmental Protection Agency 2004), SWMM is capable of simulating a dynamic model with rainfall events and a variety of watershed conditions for an urban area. SWMM takes as inputs (a) geometric data and basic hydrodynamics data to construct the river, (b) spill location and spill time of contaminant, and (c) rain location, rain starting time, rain duration and rain intensity data. For a specific river, geometric and basic hydrodynamics data are fixed but data related to contaminant spill and rainfall events contain randomness.

For process simulation, we distinguish two cases: steady state flow and dynamic flow cases. In the steady state flow case, the only randomness comes from a spill location. A spill event always occurs at the start 
time of the process simulation $\left(T_{s}=0\right)$, and there is no rainfall event. In dynamic flow case, $T_{s}$ is a random starting time of a spill event at a random location. Each possible monitoring location has its own rain event and a rain event at a node needs three random variables, rain starting time $\left(T_{R}^{i}\right)$, rain duration $\left(D_{R}^{i}\right)$, and rain intensity $\left(I_{R}^{i}\right)$.

With above randomly generated input data (a spill location, $T_{S}, T_{R}^{i}, D_{R}^{i}$, and $I_{R}^{i}$ ) and geometric information (locations, elevations and shapes at each node and reaches placed between nodes), SWMM returns concentration levels of the contaminant at each node at every constant inter-reporting time. By reading concentration level at $x_{i}$ from SWMM output, we can get $t_{d}\left(x_{i}\right)$ and thus $t(\mathbf{x})$ and $R(\mathbf{x})$.

\section{FINDING THE BEST UNDER A STOCHASTIC CONSTRAINT}

The optimization problem described in Section 2 is difficult to solve because both $\mathbf{E}[t(\mathbf{x})]$ and $\mathbf{E}[R(\mathbf{x})]$ are estimated only through simulations. Heuristic methods can be used as in Telci et al. (2008) and Telci et al. (2009) but there is no guarantee about the quality of the returned solution. Some OvS algorithms such as NP or COMPASS provide global or local convergence. However, they cannot handle stochastic constraints and thus NP or COMPASS is not directly applicable to our problem. In this section, we propose the penalized objective function that combines both the primary and secondary performance measures into one function. The new objective function enables the use of existing OvS algorithm to an optimization problem with a stochastic constraint. A brief description of the NP method with the penalized objective function is also presented.

\subsection{Penalized Objective Function}

In deterministic optimization, one way to handle constraints is to employ a penalty function where penalty is added to the original objective when a solution is infeasible (for example, see Nocedal and Wright (1999)). If Problem (4) were deterministic, then the new objective with penalty would be $\mathbf{E}[t(\mathbf{x})]+\lambda \times \max \{0, q-\mathbf{E}[R(\mathbf{x})]\}$ for a large constant $\lambda \in R^{+}$. In stochastic optimization, $\mathbf{E}[t(\mathbf{x})]$ and $\mathbf{E}[R(\mathbf{x})]$ need to be replaced with their estimates (usually sample averages), but then estimation error is inevitable and causes a risk of adding a big penalty to a feasible solution.

Many OvS algorithms repeat search iterations where a number of candidate solutions are sampled, additional observations are obtained for the solutions, and their performances are evaluated based on cumulative sample averages of all observations obtained so far. Let $\widehat{\mathbf{E}_{k}}[t(\mathbf{x})]$ and $\widehat{\mathbf{E}_{k}}[R(\mathbf{x})]$ represent estimators for $\mathbf{E}[t(\mathbf{x})]$ and $\mathbf{E}[R(\mathbf{x})]$ at search iteration $k$ based on all cumulative observations. Here the estimators are sample averages. Also define $\lambda_{k}(\mathbf{x})$ as a real-valued function which represents penalty at search iteration $k$ for solution $\mathbf{x}$. Then the penalized objective function at search iteration $k$ is defined as

$$
Z_{k}(\mathbf{x})=\widehat{\mathbf{E}_{k}}[t(\mathbf{x})]+\lambda_{k}(\mathbf{x}) \times \max \left\{0, q-\widehat{\mathbf{E}_{k}}[R(\mathbf{x})]\right\}
$$

where

$$
\begin{aligned}
& \lambda_{k}(\mathbf{x})= \begin{cases}\lambda_{k-1}(\mathbf{x}) \times \theta_{1}, & \text { if } \widehat{\mathbf{E}_{k}}[R(\mathbf{x})]<q ; \\
\lambda_{k-1}(\mathbf{x}) \times \theta_{2}, & \text { if } \widehat{\mathbf{E}_{k}}[R(\mathbf{x})] \geq q,\end{cases} \\
& \lambda_{0}(\mathbf{x})=1 \text { for all } \mathbf{x} \in \Theta, \theta_{1}>1, \text { and } \theta_{2}<1 .
\end{aligned}
$$

Note that $\lambda_{k}(\mathbf{x})$ is small at the beginning of search (small $k$ ) but converges to zero for a feasible solution and diverges to infinity for an infeasible solution. Thus $Z_{k}(\mathbf{x})$ converges to $\mathbf{E}[t(\mathbf{x})]$ for a feasible solution and diverges to infinity for an infeasible solution if the number of observations obtained up to search iteration $k$ for $\mathbf{x}$ goes to infinity as $k$ approaches infinity.

To obtain the solution to Problem (4), we solve the following problem:

$$
\operatorname{argmin}_{\mathbf{x} \in \Theta} \lim _{k \rightarrow \infty} Z_{k}(\mathbf{x}) .
$$

It is clear that the optimal solution to Problem (6) is also the best solution to Problem (4). As Problem (6) does not have stochastic constraints, any OvS algorithm can be used to solve Problem (6). In this paper, we employ the NP method as we are interested in finding a global optimal solution.

The choices of $\theta_{1}$ and $\theta_{2}$ depend on the problem characteristic and may affect the performance of an optimization algorithm. Park and Kim (2010) discuss guidelines for the choices of $\theta_{1}$ and $\theta_{2}$ and convergence properties of OvS algorithms with the penalized objective function. 


\subsection{The Nested Partitions Method with the Penalized Objective Function}

In this section, we provide the outline of the NP method with the penalized objective function. We first give the list of notation.

$\mathscr{R}_{k}:=$ the most promising region at search iteration $k$;

$\mathscr{R}_{k}(\ell):=$ the $\ell$ th subregion at search iteration $k$;

$\Theta \backslash \mathscr{R}_{k}:=$ the surrounding region at search iteration $k$;

$\mathbf{V}_{k}:=$ the set of all solutions visited up to iteration $k$;

$\mathbf{S}_{k}:=$ the set of solutions sampled at iteration $k$;

$\mathbf{x}_{k}^{*}:=$ sample best among all sampled solutions up to iteration $k$ (i.e., $\mathbf{x} \in V_{k}$ );

$\Delta n:=$ the number of observations taken from a sampled solution;

$n_{k}(\mathbf{x}):=$ the cumulative number of generated observations for $\mathbf{x}$ up to iteration $k$;

$\omega:=$ the number of subregions;

$\tau_{k}:=$ the total number of sampled solutions at iteration $k$; and

$\tau_{k}(\ell):=$ the number of sampled solutions at iteration $k$ from subregion $\ell$.

NP focuses on $\mathscr{R}_{k}$ called the most promising region and spends more computational efforts in $\mathscr{R}_{k}$. NP systematically partitions $\mathscr{R}_{k}$ into $\omega$ subregions. Then it samples and assesses solutions from the subregions and the surrounding region. If the current sample best solution is in $\mathscr{R}_{k}$, the subregion with the current sample best will be the next promising region, $\mathscr{R}_{k+1}$. Otherwise, $\Theta$ will be $\mathscr{R}_{k+1}$.

A brief implementation summary of NP with the penalized objective is provided below. For more detailed description of the NP method, see Shi and Ólafsson (2000).

\section{Algorithm : NP with Penalized Objective}

\section{Step 0. Initialization:}

- Set search iteration $k=1$ and $\mathscr{R}_{k}=\Theta$.

- Set $n_{k}(\mathbf{x})=0$ for all $\mathbf{x} \in \Theta$ and $\mathbf{V}_{0}=\emptyset$.

- Select user provided constants $\omega, \tau_{k}$, and $\Delta n$.

Step 1. Partitioning:

- If $\mathscr{R}_{k}$ is a singleton, set $M_{k}=1$. Otherwise, $M_{k}=\omega$.

- Partition $\mathscr{R}_{k}$ into $M_{k}$ disjoint regions, $\mathscr{R}_{k}(1), \mathscr{R}_{k}(2), \ldots, \mathscr{R}_{k}\left(M_{k}\right)$.

- If $\mathscr{R}_{k} \neq \Theta, M_{k}=M_{k}+1$ and $\mathscr{R}_{k}\left(M_{k}\right)=\Theta \backslash \mathscr{R}_{k}$.

Step 2. Sampling Solutions:

- From each region $\mathscr{R}_{k}(\ell), \ell=1,2, \ldots, M_{k}$, randomly sample $\tau_{k}(\ell)$ solutions. We choose $\tau_{k}(\ell)$ proportional to the size of $\mathscr{R}_{k}(\ell)$ and it satisfies $\sum_{\ell=1}^{M_{k}} \tau_{k}(\ell)=\tau_{k}$.

- Include all the sampled solutions $\mathbf{x}$ into $\mathbf{S}_{k}$.

- If $\mathbf{x} \notin \mathbf{V}_{k}$ for any $\mathbf{x} \in \mathbf{S}_{k}$, then $\mathbf{V}_{k}=\mathbf{V}_{k} \cup\{\mathbf{x}\}$.

Step 3. Estimating the Promising Index: For each $\mathbf{x} \in \mathbf{S}_{k}$,

- Take $\Delta n$ observations corresponding to the primary and secondary performance measures and set $n_{k}(\mathbf{x})=n_{k-1}(\mathbf{x})+\Delta n$.

- Update $Z_{k}(\mathbf{x})$ and select $\mathbf{x}_{k}^{*}$ such that $\mathbf{x}_{k}^{*} \equiv \operatorname{argmin}_{\mathbf{x} \in \mathbf{V}_{k}} Z_{k}(\mathbf{x})$.

Step 4. Selecting the Most Promising Region:

- Determine $\mathscr{R}_{k}\left(\ell^{*}\right)$ that contains $\mathbf{x}_{k}^{*}$.

Step 5. Backtracking:

- If $\mathscr{R}_{k}\left(\ell^{*}\right) \in \mathscr{R}_{k}$, then $\mathscr{R}_{k+1}=\mathscr{R}_{k}\left(\ell^{*}\right)$. Else, $\mathscr{R}_{k+1}=\Theta$.

- Set $k=k+1$.

Remark: See Park et al. (2010) for detailed algorithms for river node indexing, partitioning, and solution sampling for the water quality monitoring problem. The solution sampling scheme given in Park et al. (2010) ensures that $n_{k}(\mathbf{x})$ goes to infinity as $k$ increases.

Steps 1 through 5 are repeated until stopping criteria are satisfied. We use the following stopping criteria similar to those in Pichitlamken and Nelson (2003).

\section{Stopping Criteria}

Let $E_{1}$ be an event such that

$$
E_{1}:=\left\{\mathbf{x}_{k}^{*}=\mathbf{x}_{k-1}^{*},\left|Z_{k}\left(\mathbf{x}_{k}^{*}\right)-Z_{k-1}\left(\mathbf{x}_{k-1}^{*}\right)\right|<\varepsilon, \mathscr{R}(k) \text { is a singleton }\right\}
$$




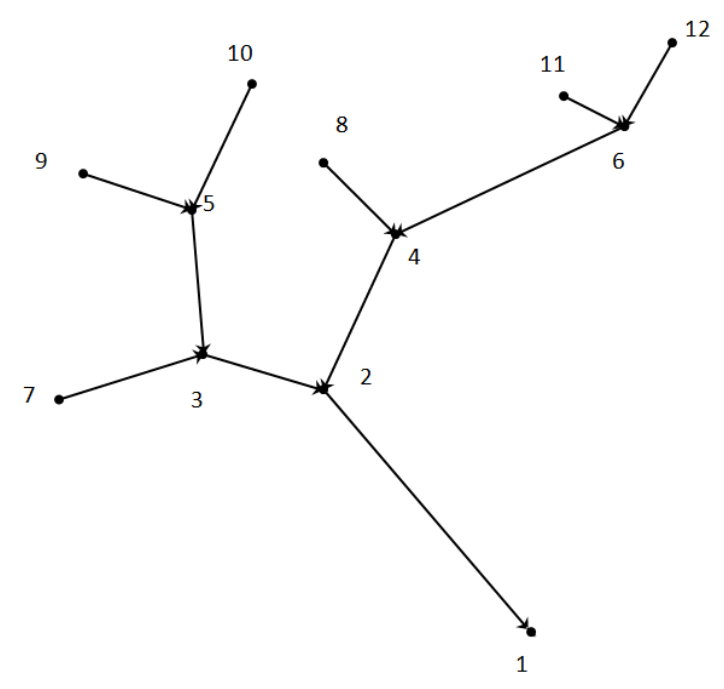

Figure 1: Shape of the hypothetical river and possible monitoring locations

where $\varepsilon$ is a small constant. We set $\varepsilon=0.1$ and the algorithm terminates if $E_{1}$ occurs 10 times consecutively.

\section{EXPERIMENTAL RESULT}

In this section, we discuss experimental set up and results. We test the performance of NP with the penalized objective on the hypothetical river from Ouyang et al. (2008). The hypothetical river has 12 possible monitoring locations $(N=12)$. Figure 1 shows a shape, flow direction and possible monitoring locations of the hypothetical river.

We assume that reliability should be at least $80 \%$ (i.e, $q=0.8$ ). There is only one spill at one location during whole simulation run length and a spill event occurs at node $i$ with probability $1 / N$. The number of identical monitoring devices is three $(M=3)$ and a device detects a spill if contaminant concentration is equal to or greater than $0.0001 \mathrm{mg} / \ell$ (i.e., $C_{t h}=0.0001 \mathrm{mg} / \ell$ ). Total simulation run length is 24 hours (1 day) for each process simulation $P_{v}=7$ hours which is longer than 214 minutes, the longest travel time of a contaminant in the hypothetical river.

The completion time of a SWMM run is about 20 seconds for the hypothetical river but it can be couple of hours for a large river when implemented on an IMB PC with $2.5 \mathrm{GHz}$ CPU. Also reading SWMM output to get $t(\mathbf{x})$ and $R(\mathbf{x})$ can take a couple of minutes on the same machine. To save computational time for running SWMM, we use common random numbers across solutions, which result in the same contaminant spill and rainfall events across solutions. This implies that all solutions have the same inputs for SWMM and the same SWMM output will be obtained. Thus each SWMM output can be shared by all sampled solutions at iteration $k$ and we only need to read contaminant concentration levels at different monitoring locations $\mathbf{x}$ to obtain $t(\mathbf{x})$ and $R(\mathbf{x})$.

For the penalized objective function, we set $\theta_{1}=2$ and $\theta_{2}=0.1$. These values are chosen based on some simple experiments but more discussion about the choices of the parameters is in Park and Kim (2010) and Park et al. (2010). For NP, we use $\omega=2, \tau_{k}=5$, and $\Delta n=2$. Due to computation time, we made only 10 macro replications.

\subsection{Steady State Flow}

For the steady state flow case, a spill location is the only random factor. The spill event occurs at the beginning of the simulation and there is no rainfall event. This case is considered in Telci et al. (2008) with 15 minutes inter-reporting time for SWMM.

As there are only 220 solutions and 12 possible random events (a spill event occurs at node $i=1,2, \ldots, 12$ with equal probability) for a given solution $\mathbf{x}$, they obtained the true value of $\mathbf{E}[t(\mathbf{x})]$ and $\mathbf{E}[R(\mathbf{x})]$ by running all 12 random events (i.e., 12 SWMM runs) for all possible $\mathbf{x}$. They found $(1,2,6)$ as the best solution. 


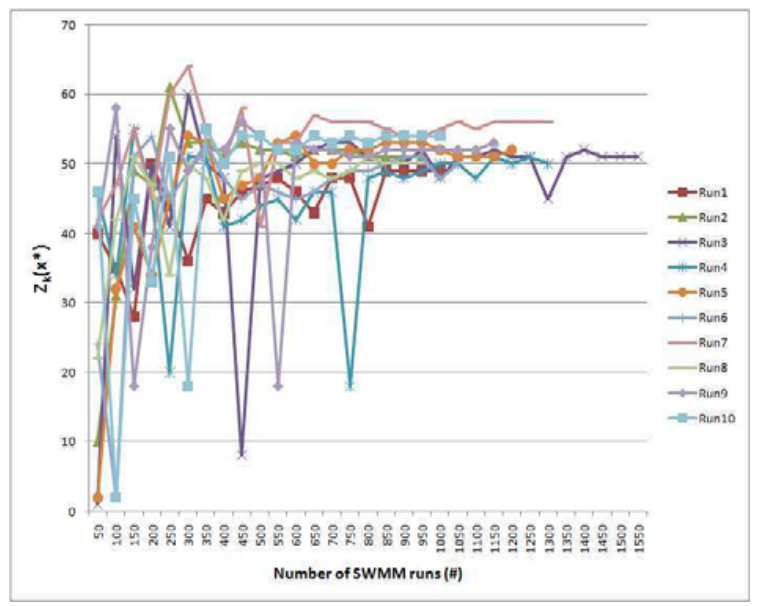

Figure 2: Realization of $Z_{k}\left(\mathbf{x}^{*}\right)$ in the dynamic flow case

The expected minimum detection time $(\mathbf{E}[t(1,2,6)])$ and reliability $(\mathbf{E}[R(1,2,6)])$ were 63.75 minutes and $100 \%$. Given that the best solution has the expected minimum detection time of 63.75 minutes, the 15 -minute inter-reporting time is somewhat large. When we reduced the inter-reporting time to 2 minutes, $(1,2,6)$ was still the best solution with $\mathbf{E}[t(1,2,6)]=45$ minutes and $(1,3,4)$ was the second best solution with $\mathbf{E}[t(1,3,4)]=45.5$ minutes. Both had $100 \%$ reliability.

As the numbers of solutions and random events are small, an OvS algorithm is not needed for the steady state case. However, the steady state case gives some ideas about solutions that are expected to perform well. In next subsection, we consider the dynamic flow case and apply NP with the penalized objective function.

\subsection{Dynamic Flow Case}

In the dynamic flow case, we assume that $T_{S}$ is uniformly distributed between 0 and 12 (hours) at a random spill location. For rainfall events, $T_{R}^{i}, D_{R}^{i}$ and $I_{R}^{i}$ are assumed to be uniformly distributed between 0 and 20 (hours), 0 and 3 (hours) and 0 and 4 (inch/hour), respectively. For a given $\mathbf{x}$, the number of random events is infinity due to continuous random variables for spill time and rainfall events and thus the true expected minimum detection time and reliability can not be obtained by a finite number of SWMM runs unlike the steady state flow case.

NP with the penalized objective was applied to the dynamic flow case and Figure 2 shows 10 realizations of $Z_{k}\left(\mathbf{x}^{*}\right)$. As shown in Table 1, NP returned $(1,2,6),(1,3,4)$ or $(1,3,6)$ as the best solution in 10 macro replications. Estimated reliability was $100 \%$ for all three solutions. Since $(1,2,6),(1,3,4)$, and $(1,3,6)$ were returned as the final solutions, 5000 SWMM runs were made for the three solutions. Table 2 shows sample average and standard deviation of minimum detection times, $t(\mathbf{x})$ from 5000 SWMM runs for the three solutions. Solution $(1,2,6)$ has slightly smaller sample average minimum detection time but $(1,3,4)$ has smaller standard deviation.

We also calculated simultaneous confidence intervals for the difference between each pair of the three solutions with overall confidence level $95 \%$. Thus each confidence interval for the difference in Table 3 was constructed at $98.3 \%$ confidence level. The results in the table imply that $(1,2,6)$ and $(1,3,4)$ are likely to be better than $(1,3,6)$. Solution $(1,3,4)$ has slightly higher sample average minimum detection time than $(1,2,6)$ but the difference is very small (around 1 minute) and $(1,3,4)$ has smaller variability. Thus $(1,3$, 4) seems better than $(1,2,6)$. NP with the penalized objective function also chose $(1,3,4)$ as the best most often.

\section{DISCUSSION}

We propose the penalized objective function that integrates a stochastic constraint into the original objective function and enables the use of an existing OvS algorithm to an optimization problem with a stochastic constraint. Although we proposed the method for the water quality monitoring problem, it is applicable to general stochastic optimization with a stochastic constraint. The performance of NP with the penalized objective is tested on a hypothetical river. Experimental results show that the method works well. Application 
Table 1: Results of NP with the penalized objective function at termination in the dynamic flow case

\begin{tabular}{||c||c|c|c|}
\hline $\begin{array}{c}\text { Macro } \\
\text { Replication }\end{array}$ & $\begin{array}{c}Z_{k}\left(\mathbf{x}^{*}\right) \\
(\mathrm{min})\end{array}$ & $\begin{array}{c}\text { Returned Solution } \\
\mathbf{x}^{*}\end{array}$ & $\begin{array}{c}\text { Total number of } \\
\text { SWMM Runs }\end{array}$ \\
\hline \hline Rep 1 & 49.02 & $(1,2,6)$ & 1032 \\
\hline Rep 2 & 50.80 & $(1,3,4)$ & 974 \\
\hline Rep 3 & 51.08 & $(1,3,4)$ & 1570 \\
\hline Rep 4 & 50.18 & $(1,2,6)$ & 1332 \\
\hline Rep 5 & 51.84 & $(1,3,4)$ & 1240 \\
\hline Rep 6 & 50.21 & $(1,2,6)$ & 1062 \\
\hline Rep 7 & 56.19 & $(1,3,6)$ & 1336 \\
\hline Rep 8 & 50.19 & $(1,3,4)$ & 910 \\
\hline Rep 9 & 52.32 & $(1,3,4)$ & 1166 \\
\hline Rep 10 & 53.59 & $(1,3,4)$ & 1034 \\
\hline
\end{tabular}

Table 2: Statistics for three solutions based on 5000 SWMM runs in the dynamic flow case

\begin{tabular}{||c||c|c|c||}
\hline Solution & $(1,2,6)$ & $(1,3,4)$ & $(1,3,6)$ \\
\hline \hline Average Minimum Detection Time & 51.3288 & 52.5912 & 54.5464 \\
\hline Standard Deviation of Minimum Detection time & 45.5454 & 37.0991 & 49.7081 \\
\hline Upper bound of a 95\% C.I. for $E[t(\mathbf{x})]$ & 50.0663 & 51.5629 & 53.1686 \\
\hline Lower bound of a 95\% C.I. for $E[t(\mathbf{x})]$ & 52.5911 & 53.6195 & 55.9242 \\
\hline
\end{tabular}

Table 3: Simultaneous confidence intervals for three solutions based on 5000 SWMM runs in the dynamic flow case

\begin{tabular}{||c|c||}
\hline & $98.3 \%$ Confidence Interval \\
\hline $\mathbf{E}[t(1,2,6)]-\mathbf{E}[t(1,3,4)]$ & {$[0.26,-2.79]$} \\
\hline $\mathbf{E}[t(1,3,4)]-\mathbf{E}[t(1,3,6)]$ & {$[-0.09,-3.82]$} \\
\hline $\mathbf{E}[t(1,2,6)]-\mathbf{E}[t(1,3,6)]$ & {$[-1.95,-4.48]$} \\
\hline
\end{tabular}

of the method to a real river is discussed in Park et al. (2010). Park and Kim (2010) discuss convergence of NP with the penalized objective and other implementation issues. Extension to multiple stochastic constraints is the subject of ongoing research.

\section{ACKNOWLEDGMENTS}

This work is supported by NSF grant number CMMI-0644837.

\section{REFERENCES}

Beckers, V., and G. Chamberlain. 1974. Design of cost-effective water quality surveillance systems. Technical Report No. 428, Raytheon Company, Oceanographic and Environmental Services, Portsmouth.

Esterby, S., A. El-Shaarawi, and H. Block. 1992. Detection of water quality changes along a river system. Environmental Monitoring and Assessment 23 (1): 219-242.

Hong, L., and B. Nelson. 2006. Discrete optimization via simulation using COMPASS. Operations Research 54 (1): 115-129.

Kwiatkowski, R. 1991. Statistical needs in national water quality monitoring programs. Environmental Monitoring and Assessment 17 (2): 253-271.

Nocedal, J., and S. Wright. 1999. Numerical optimization. Springer: New York.

Ouyang, H., H. Yu, C. Lu, and Y. Luo. 2008. Design Optimization of River Sampling Network Using Genetic Algorithms. Journal of Water Resources Planning and Management 134:83-87.

Park, C., and S.-H. Kim. 2010. Discrete optimization with stochastic constrains via simulation. Technical Report, Georgia Institute of Technology, Atlanta, Georgia. 
Park, C., I. Telci, M. M. Aral, and S.-H. Kim. 2010. Designing optimal water quality monitoring network for river systems with the optimization via simulation method. Technical Report, Georgia Institute of Technology, Atlanta, Georgia.

Pichitlamken, J., and B. Nelson. 2003. A combined procedure for optimization via simulation. ACM Transactions on Modeling and Computer Simulation 13 (2): 155-179.

Shi, L., and S. Ólafsson. 2000. Nested partitions method for stochastic optimization. Methodology and Computing in Applied Probability 2: 271-291.

Skalski, J., and D. McKenzie. 1982. A design for aquatic monitoring programs. Journal of Environmental Management 14 (3): 237-251.

Telci, I., K. Nam, J. Guan, and M. Aral. 2008. Real time optimal monitoring network design in river networks. In World Environmental and Water Resources Congress 2008, ed. R. Babcock and R. Walton, 1-10. Honolulu, Hawaii: Environmental And Water Resources Institute (EWRI) of the American Society of Civil Engineers.

Telci, I., K. Nam, J. Guan, and M. Aral. 2009. Optimal water quality monitoring network design for river systems. Journal of environmental management 90:2987-2998.

U.S. Environmental Protection Agency 2004. Storm water management model users manual version 5.0. 5th ed. Cincinnati: U.S. Environmental Protection Agency.

\section{AUTHOR BIOGRAPHIES}

CHULJIN PARK is a Ph.D. student in the H. Milton Stewart School of Industrial and Systems Engineering at the Georgia Institute of Technology. He received his B.S. in Mechanical Engineering from Korea Advanced Institute of Science and Technology (KAIST) in 2007. His research interests include optimization via simulation with stochastic constraints with applications to environmental management. His email address is <cpark41@mail.gatech.edu>.

SEONG-HEE KIM is an Associate Professor in the H. Milton Stewart School of Industrial and Systems Engineering at the Georgia Institute of Technology. She received her Ph.D. in Industrial Engineering and Management Sciences from Northwestern University in 2001. Her research interests include ranking and selection, optimization via simulation, quality control, simulation output analysis, and applications of simulation methods to environmental management. She is a member of INFORMS, and serves as an associate editor in the simulation area of Operations Research and the OR/simulation area of The American Statistician. She also served on the editorial board of the Simulation Department of IIE Transactions. Her e-mail and web addresses are<skim@isye.gatech.edu> and <www. isye.gatech.edu/skim/> respectively.

ILKER T. TELCI is a Ph.D. student in the School of Civil and Environmental Engineering at the Georgia Institute of Technology. He received his B.S. and M.S. degrees in Civil Engineering from Middle East Technical University (METU), Ankara, Turkey in 1999 and 2002, respectively. His research interests include optimization and classification and pattern recognition methods in environmental engineering applications. His email address is <itelci3@mail.gatech.edu>.

MUSTAFA M. ARAL is a Professor in the School of Civil and Environmental Engineering at the Georgia Institute of Technology. His research interests include large scale environmental simulations in surface and ground water, environmental exposure analysis, exposure-dose reconstruction, and health risk assessment. His e-mail and web addresses are <maral@ce.gatech.edu> and <http://mesl.ce.gatech.edu/Aral/>, respectively. 